\title{
Leitartikel
}

\section{Ethik des Rennsports - eine Herausforderung für die Branche, aber auch eine Chance}

\author{
Dagmar Borchers \\ Lehrstuhl für Angewandte Philosophie, Universität Bremen, Bremen
}

\section{Einleitung}

Der Rennsport fasziniert viele Menschen - nicht nur jene, die als Züchter, Trainer, Besitzer oder Wettende involviert sind. Die Schönheit und Eleganz der Pferde, die Spannung bei den Rennen und die großartige Athmosphäre auf dem Rennplatz motivieren viele Pferdeliebhaber und auch zunehmend Familien dazu, Nachmittage auf dem Rennplatz zu verbringen. Neben jenen, die im Kontext einer Wette auch ein gewisses materielles Interesse am Wettkampf haben, sind es vor allem diese Pferdefreunde, die aus reiner Freude am Geschehen und aus Liebe zu den Tieren dabei sein wollen und so die Zukunft des Rennsports maßgeblich mitgestalten und vielleicht auch langfristig sichern werden.

Der Rennsport kann auf eine lange, historisch interessante und von vielen Traditionen geprägte Geschichte zurückblicken, die die heutige Praxis immer noch prägt und viel zu deren Charme und besonderer Ausstrahlung beiträgt. Aber der gesellschaftliche Kontext, in dem der Pferderennsport stattfindet, hat sich stark verändert: Auf der einen Seite kann man feststellen, dass ökonomische Aspekte stärker denn je dominieren, denn es ist eine globale Rennsportindustrie involviert. Auf der anderen Seite verzeichnet der Tierschutz und die Frage nach dem Wohlergehen der Tiere eine zunehmende Relevanz und auch Brisanz: Viele Menschen sind heute für diese Frage hoch sensibilisiert und in und für den Tierschutz engagiert. Auch in dieser Hinsicht rückt der Rennsport zunehmend in den Fokus der Öffentlichkeit. Der Status der Ethik bzw. des Tierschutzes hat sich in den letzten Jahren stark verändert. Ethik ist nicht mehr eine, nette Nebensache', über die man gelegentlich einmal reden kann - im Gegenteil: die Frage, was mit den Pferden im Rennsport passiert, wie sie gezüchtet, gehalten und trainiert werden, welchen Risiken sie im Rennen ausgesetz† sind, wie viele sich verletzen oder im Rennen sterben und was nach ihrer Laufbahn als Rennpferd mit den Tieren geschieht, werden mehr und mehr zu den entscheidenden Fragen, die von Seiten der Öffentlichkeit an den Rennsport gestellt werden. Die Tatsache, dass sich der Rennsport selbst als ein globales Geschäft bzw. eine Industrie versteht, suspendiert ihn dabei keineswegs davon, diese ethischen Aspekte ernst zu nehmen und überzeugende Antworten auf diese Fragen zu entwickeln - Antworten, hinter denen eine ethisch akzeptable Praxis steht.

Der Rennsport steht also vor der Herausforderung, sich ernsthaft und intensiv mit der Frage des Wohlergehens der Pferde zu beschäftigen. Das ist inhaltlich und organisatorisch keine Kleinigkeit. Es bedeutet zunächst einmal, eine (selbst-)kritische Bestandsaufnahme durchführen, den eigenen Blick auf das Geschäft zu schärfen und dann Vorschläge zu entwickeln, wie man Missstände beheben und die Praxis des Rennsports nachhaltig verändern kann. Am schwierigsten wird dann jedoch die konkrete Umsetzung werden - auch dies wird sich als eine Herausforderung eigener Art erweisen.

Dringend gefragt ist also eine Ethik des Rennsports. Ich möchte dazu einen Vorschlag machen, der mehr umfasst als ein paar singuläre Verbesserungsvorschläge. Meine Idee ist, ein umfassendes Ethik-Konzept zu entwickeln, das dem Rennsport zugleich ein neues, auch für die Außendarstellung gut geeignetes Selbstverständnis und einen kohärenten, theoretisch bzw. tierethisch fundierten Rahmen für die unterschiedlichen Einzelmaßnahmen anbietet.

\section{Ethisch handeln, rational handeln - das Dilemma des Einzelnen}

Was genau wird kritisiert? Zunächst einmal sind es natürlich die Risiken, denen die Tiere im Rennen selbst ausgesetz† sind. Durch die Medien, aber auch durch den engagierten Tierschutz finden Bilder von schwer verwundeten oder sterbenden gestürzten Pferden Verbreitung und schockieren viele Menschen. Bilder dieser Art sind dann gewissermaßen der Anstoß weiter zu fragen: Wie werden die Pferde gehalten? Wie werden sie trainiert? Welche Rolle spielen Medikamente und Doping? In welchem Alter beginnen die Pferde mit den Rennen? Und was passiert, wenn sie nicht mehr die erwartete Leistung bringen können? Dies sind bisher die wichtigsten Kritikpunkte:

- Der Einsatz von Medikamenten und Medikamentenmissbrauch im Sinne von Doping.

- Die Pferde laufen ihr erstes Rennen zu früh.

- Die Pferde werden nicht artgerecht gehalten; sie stehen in dunklen Ställen, kommen nicht genug auf die Weise und haben zu wenig Kontakt zu Artgenossen.

- Die Pferde sind auf dem Rennplatz auch schon vor dem Rennen extremem Stress ausgesetzt, u. a. deshalb, weil sie nicht gut genug vorbereitet werden. So kommt es immer wieder zu Unfällen und unangenehmen Vorfällen an der Startmaschine. 
- Die Pferde werden rigoros ausgemustert, wenn sie die erwartete Leistung nicht bringen. Viele gehen zum Schlachter oder leben unter sehr schlechten Bedingungen.

Man könnte noch weitere Punkte hinzufügen: Etwa die Strapazen, die die ständigen (weltweiten) Transporte mit sich bringen oder der Einsatz von Peitschen im Rennen. Generell hat sich bei vielen Beobachtern des Rennsports der Eindruck verfestigt, dass die Tiere im Rahmen einer hoch professionell arbeitenden Optimierungsmaschinerie, in der neben Züchtern, Trainern und Besitzern auch Tierärzte eine zentrale Rolle spielen, ein kurzes, völlig artunangemessenes Leben führen und dann aussortiert werden. Sie werden als Produkt einer globalen Industrie hergestellt und benutzt wie Sportartikel.

Dieses Bild, so möchte man hoffen, ist überspitzt und in weiten Teilen unzutreffend. Was kann der Rennsport tun, um diesen Vorwürfen entgegenzutreten?

Wer über Ethik redet, sollte realistisch sein. Deshalb sollte man sich zunächst vor Augen führen, warum es für den einzelnen so schwer ist, das Verhalten zu ändern und die ethischen Standards zu verbessern. Man kann dies besonders deutlich am Beispiel des Einsatzes von Medikamenten im Sport aufzeigen: Medikamentenmissbrauch im Sinne von Doping gibt es in vielen Sportarten. Oft sind die Übergänge fließend zwischen dem, was, noch erlaubt' ist und sich rechtlich und ethisch in einer Grauzone befindet und dem, was nicht mehr statthaft ist. Im Pferderennsport haben wir es mit einer globalen Industrie zu tun, in der Züchter, Trainer, Beisitzer, Tierärzte und diejenigen, die Wetten abschließen ökonomisch profitieren wollen. Sie alle haben auch massive kommerzielle Interessen und stehen unter hohem Erfolgsdruck. Konkurrenz und Wettbewerb herrschen nicht nur im Rennen, also im sportlichen Wettkampf selbst, sondern auch im Vor- und Umfeld, im Bereich der Züchtung, Betreuung und des Trainings. Dies ist nun in der Tat ein extrem moralfeindliches Umfeld - denn in jedem Kontext, in dem Gewinnmaximierung, Profit und Konkurrenz dominieren, scheinen Kooperation und Fairness aus der Perspektive der Akteure nicht rational zu sein. Es ist nicht vernünftig, sich fair und moralisch zu verhalten, wenn man davon ausgehen muss, dass alle anderen es nicht tun. In einem solchen Umfeld besteht stets die Gefahr, dass Ehrlichkeit und Moralität ausgebeutet und ausgenutzt werden. Die ,Logik des Systems' generiert eine sog. "Rationalitäłsfalle", die spieltheoretisch als "Gefangendilemma" bezeichnet wird (Behnke 2013, Diekmann 2009). Dies kann zu einer Situation führen, in der diejenigen, die die Pferde möglichst effizient nutzen und dabei möglichst wenig Rücksicht nehmen, zu den Erfolgreichsten gehören. Solange die Bekämpfung von Doping und anderen Missständen nicht eine Angelegenheit des Gesamtsystems selbst wird, besteht für den einzelnen, der weiterhin erfolgreich sein will, wenig Anreiz, sein Verhalten zu ändern, sofern er rational handelt. Das Dilemma, das sich für den einzelnen vor diesem Hintergrund ergibt, sieht folgendermaßen aus:

Ist man als Beteiligter bereit, (hohe) moralische Standards einzuhalten, besteht die reale Gefahr, dass diese Bereitschaft ausgenutzt wird und man weniger erfolgreich agiert. Ist man nicht bereit, moralische Standards einzuhalten, ist man Teil eines Systems, das sich zunehmend selbst untergräbt, und es besteht die reale Gefahr, dass man nicht nur sich selbst, sondern auch dem Rennsport langfristig massiven Schaden zufügt.

Moral zu predigen, bringt daher m. E. eher wenig. Appellen hört man gerne zu, aber sie wirken in der alltäglichen Praxis oftmals wenig nach. Als Ethikerin sollte man sich vielmehr fragen: Was könnten mögliche Anreize für das System als Ganzes sein, unmoralische Praktiken (sei es exzessiver Gebrauch von Medikamenten oder andere Maßnahmen, die das Tierwohl ignorieren) aufzugeben und ggf. massiv in ein neues Selbstverständnis und modifizierte interne Regeln zu investieren? Von "Anreizen" zu sprechen bedeutet, dass man nicht davon ausgeht, dass Verhaltensänderungen notwendigerweise von moralischen Motiven bei den Akteuren induziert werden. Es könnten auch Klugheitserwägungen sein, also Gründe, denen zu folgen aus (langfristigem) Selbstinteresse geboten zu sein scheint. Ob man dann überhaupt noch von einer moralischen Handlung sprechen kann, ist innerhalb der Moralphilosophie umstritten. Während für einen Pflichtethiker (wie zum Beispiel Immanuel Kant) eine Handlung nur dann moralisch ist, wenn sie aus ethischen Motiven heraus geschieht (bei Kant deshalb, weil man erkennt, dass diese Handlung eine moralische Pflicht ist), betonen Konsequentialisten, dass die moralische Qualität einer Handlung nicht am Handlungsmotiv, sondern an ihren Konsequenzen hängt. Aus Selbstinteresse zu handeln, ist dann nicht verwerflich, wenn aus der Perspektive des moralischen Akteurs zu erwarten ist, dass die Wahl einer Handlungsalternative für die von der Handlung Betroffenen die bestmöglichen Konsequenzen mit sich führen wird. Breit diskutierte Grundlagen des Dopingverbotes sind u. a.

- der Tierschutz;

- der sportethische Grundsatz eines fairen Wettkampfes; die Gewährleistung gleicher Voraussetzungen für alle Wettkampfteilnehmer;

- der Schutz anderer Wettkampfteilnehmer vor Gefahren, die von gedopten Tieren ausgehen können;

- die Verhinderung falscher Zuchtauslese durch Vortäuschung eines unzutreffenden Leistungsstandes des Pferdes;

- der Schutz vor Täuschung des zahlenden Publikums, das entscheidend zur Förderung des Pferdesports beiträgt sowie

- eine mögliche strafrechtliche Relevanz.

Die beiden ersten Gründe kann man als ethisch bezeichnen, die folgenden vier sind eher rationale Gründe. Wollte man diese Diskussion um rationale Anreize für moralisches Handeln vertiefen, wäre im einzelnen aufzuzeigen, inwiefern Medikamentenmissbrauch und andere unfaire Praktiken im Rennsport eine negative Konkurrenz verursachen, die ihrerseits zu einer Verschärfung der Situation, nämlich einer Ausweitung dieser Praktiken und deren substanzieller Verankerung im Rennsport führen würden. Es wäre der Nachweis zu führen, dass sich dies negativ auf das Interesse der Zuschauer sowie der Öffentlichkeit auswirken würde und es wäre schließlich zu erkennbar zu machen, dass sich der Rennsport damit selbst massiv schaden würde. All dies müsste auf die 
Prognose hinauslaufen: Das System scheitert schließlich an seiner eigenen, internen (negativen) Logik. Es gerät zunehmend in Konflikt mit denjenigen, die für den Tierschutz eintreten, die an einem artgerechten und fairen Rennsport interessiert sind und die als Beteiligte ihren eigenen moralischen Maßstäben gerecht werden möchten.

Diese Diskussion zeigt: Es geht bei der Diskussion über Ethik im Rennsport um mehr, als nur um ein wenig Moral-Kosmetik, um die leicht beunruhigte Öffentlichkeit zufrieden zu stellen. Es geht m. E. um das Selbstverständnis und die Zukunft des Rennsports, also um die Fragen: Wie sehen wir uns selbst? Welche Maßstäbe legen wir an unser Handeln an? Wie soll sich der Rennsport entwickeln? Wie stellen wir uns die Zukunft unseres Sports vor? Wie können wir adäquat und überzeugend auf das Faktum reagieren, dass das Tierwohl in unserer Gesellschaft ein hohes Gut darstellt, dass Tiere nach dem Verständnis der meisten Menschen moralisch relevant sind und dass dies wiederum bedeutet, dass wir genau überlegen und ggf. auch rechtfertigen müssen, was wir ihnen zumuten und wie wir sie behandeln? Also sprechen wir doch einmal über (Tier-)Ethik.

\section{Über Ethik}

Zu den Grundsatzdebatten in der zeitgenössischen Tierethik zählt insbesondere die Diskussion um den moralischen Status der Tiere. Mögen auch die Begründungsstrategien divergieren, so herrscht doch weitgehender Konsens darüber, dass Tiere moralisch relevant sind und wir mithin verpflichtet sind, bestimmte Standards einzuhalten und unser Verhalten gegenüber Tieren zu rechtfertigen. Natürlich gibt es innerhalb der Tierethik große Unterschiede in Bezug auf die Frage, wozu genau wir moralisch verpflichtet sind und welche Praktiken im Umgang mit Tieren unterschiedlicher Art (Haustiere, Nutztiere, Wildtiere) jeweils moralisch erlaubt sind und welche nicht.

Einen durchaus kontrovers diskutierten, aber als theoretische Fundierung für eine Ethik des Rennsports möglicherweise gut geeigneten Ansatz hat die amerikanische Philosophin Martha Nussbaum vorgelegt. Vor dem Hintergrund eines aristotelischen, philosophiehistorisch auf die Antike zurückgehenden Moralverständnisses plädiert sie für einen sog. "Fähigkeiten-Ansatz", der für verschiedene Spezies funktionieren soll. Grundsätzlich bedeutet moralisch zu handeln dieser Auffassung zufolge, auf der Basis von Mitleid und Respekt diejenigen Gegebenheiten anzuerkennen, die eine bestimmte Spezies braucht, um ihre artspezifischen Fähigkeiten und Anlagen entfalten zu können und um auf dieser Basis ein artspezifisch gutes Leben führen zu können. Für Menschen sind hier naturgemäß andere Aspekte zu berücksichtigen als für Tiere - so ist es für Menschen unerlässlich, freien Gebrauch von ihrer Vernunft machen und ihr Leben nach eigenem Ermessen gestalten zu können. Es gibt aber auch Aspekte, die Mensch und Tier verbinden - die Möglichkeit, elementare Bedürfnisse wie Hunger, Durst, Angstfreiheit und soziale Nähe befriedigen zu können, das Bedürfnis jeweils artspezifische Freiheitsspielräume in Anspruch nehmen zu können und insgesamt so zu leben, wie es für das jeweilige Tier bzw. den Menschen angemessen ist, damit die jeweiligen Fähigkeit zur Entfaltung kommen können. Die natürliche Lebensspanne möglichst auskosten zu können, produktiv sein zu können im Rahmen der naturgemäß gegebenen Möglichkeiten und Grenzen, mit Artgenossen zusammen sein zu können und dabei auch Bedürfnissen wie Spiel, Bewegung und der Herstellung von sozialen Kontakten nachgehen zu können, gehören dazu. Es gibt, so Nussbaum (2014), prima facie keinen vernünftigen Grund, Tieren diese Bedingungen zu verweigern; moralisches Handeln besteht darin, sie ihnen zuzugestehen und in diesem Sinne für sie zu sorgen.

Diese Konzeption ist eine mögliche Variante einer Tierethik, die es uns erlauben würde, einen theoretisch fundierten, inhaltlich recht ergiebigen Rahmen abzustecken, um die für eine Ethik des Rennsports zentrale Frage zu beantworten: Wie können wir den Rennsport moralisch verantwortungsvoll, in Respekt vor dem Pferd so gestalten, dass wir ihm ein artgerechtes Leben im Sinne des Fähigkeitenansatzes ermöglichen und es in diesem Rahmen gleichwohl als Rennpferd einsetzen? Ist das möglich? Lässt sich das kombinieren? Und wie würde das konkret aussehen? Es würde im Detail bedeuten, systematisch alle Stationen des Rennpferdelebens daraufhin zu untersuchen, wie man sie so gestalten kann, dass das Rennpferd im Rahmen seiner natürlichen Fähigkeiten eingesetz† wird und ein möglichst gutes, artgerechtes Leben führen kann.

Ansätze dazu gibt es bereits. Viele Tierärzte, Züchter, Tierhalter und Trainer versuchen, diesen Weg zu bestreiten und zu formulieren, was ein respektvoller Umgang mit dem Pferd, der dessen moralische Relevanz wirklich ernst nimmt, im Alltag des Rennsports verlangt. Klar ist aber auch, dass hier ein großes Problem lavert: Wer das Pferd wirklich moralisch respektiert, muss auch dessen natürliche Grenzen respektieren. Und das bedeutet ganz konkret, dass bestimmte Dinge zu unterlassen sind - exzessiver Einsatz von Medikamenten, jede Form von Doping, Überforderung im Training und im Wettkampf, zu häufiger Einsatz, etc. Hier liegt m. E. die der harte Kern der Ethik-Debatte, der schwierige Punkt: Wenn wir ernsthaft über Ethik im Rennsport diskutieren, müssen wir bereit sein, Grenzen anzuerkennen und Dinge zu unterlassen, die diese Grenzen überschreiten. Darüber muss Einigkeit unter den Beteiligten im Rennsport herrschen, dies kann nicht nur Sache des guten Willens einzelner sein - aus oben geschilderten, systematischen Gründen. Der Rennsport braucht ein neves Selbstverständnis.

\section{Ein Vorschlag: ein neues Selbstverständnis}

Basierend auf den oben ausgeführten Überlegungen scheint es mir ein sinnvolles Unterfangen zu sein, den Rennsport systematisch neu zu positionieren - nach innen, in Hinblick auf das Selbstverständnis und nach außen, in Hinblick auf die Außendarstellung in der Öffentlichkeit. Diese Idee läuft darauf hinaus, ein neues Selbstportrait zu entwerfen. Man kann es inhaltlich als eine "Re- 
Naturalisierung des Rennsports" bezeichnen. Einem Rennen beizuwohnen heißt dabei im Kern, Pferde bei etwas zu beobachten, was sie gerne tun, was ihrer Natur entspricht und bei dem ihre Eleganz und ihre Schönheit in besonderer Art zur Geltung kommen können. Zucht, Ausbildung und Sport finden innerhalb der Grenzen ihrer natürlichen Fähigkeiten statt, respektieren diese und berücksichtigen sie. Programmatisches Ziel dieser "fair-trained"-Ausrichtung ist die (Re-)Harmonisierung von Natur (des Pferdes) und Kultur (des Rennsports und seiner Traditionen. Die Menschen erfreven sich dabei an einem Vorgang, bei dem das Pferd seine natürliche Substanz voll ausschöpft und nicht der erzwungenen, bis in letzte ausgereizten Vorstellung einer genetisch perfektionierten, medikamentös maximal eingestellten Rennmaschine. Das Schaubild markiert noch einmal die Grundidee:

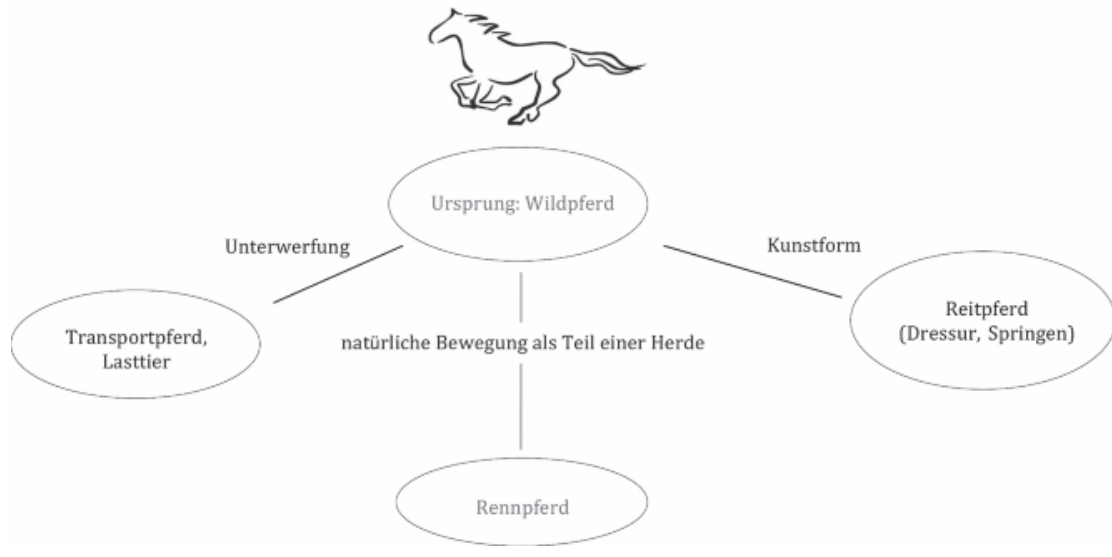

Abb. 1 Ein neues Paradigma für den Rennsport
Der Vorschlag ist, mit einer solchen Grundidee an die weitere Debatte um Ethik im Rennsport heranzugehen. Auch wenn man nicht erwarten kann, in absehbarer Zeit einen Konsens oder auch nur eine Meinungskonvergenz in diese Richtung erreichen zu können, braucht es für eine langfristig angelegte Debatte meiner Einschätzung nach eine gemeinsame Vorstellung, eine inhaltliche Ausrichtung, die die interne Diskussion strukturiert, einzelne Überlegungen in einem inhaltlichen Rahmen zusammenfasst und die Ausarbeitung einer Programmatik ermöglichst, in der Kreativität und Phantasie zum Einsatz kommen können. Vielleicht kommt man auch in Einzelschritten zu einem gemeinsamen Ziel. Grundsätzlich ist nicht entscheidend, wie die Diskussion verlaufen wird, sondern dass sie produktiv fortgesetz† wird. In der Ethik-Debatte auch das Selbstverständnis mit zu reflektieren, soll einen weiteren Anreiz darstellen, diese Debatte überhaupt zu führen und zugleich deutlich machen, welch ein inhaltliches und strategisches Potential in einer EthikDebatte liegen könnte. Der Rennsport will attraktiv bleiben, er möchte möglichst viele Zuschauer gewinnen und er möchte nicht länger im Zwielicht der zunehmenden Anklagen von Tierschützern und Medien stehen. Täuschen lassen wird sich die Öffentlichkeit aber nicht. Nur wenn wirklich eine ernsthafte Auseinandersetzung der Beteiligten erkennbar ist, deren Resultate in der Praxis erkennbar sind, wird der Rennsport überzeugen können.

\section{Abschließende Bemerkungen}

Doping, verletzte und tote Pferde und schlechte, die Bedürfnisse des Tieres ignorierende Haltungs- und Trainingsbedingungen bringen den Rennsport in Misskredit und werden ihm langfristig schaden, denn Tierschutz ist in der Öffentlichkeit ein hohes Gut. Die Öffentlichkeit ist nicht länger bereit, Ignoranz und Grausamkeit hinzunehmen und zu tolerieren. Für individuelle Besitzer, Züchter, Trainer und Tierärzte ist es schwierig, moralische Maßstäbe in einem System zu etablieren, in dem eine interne Logik die Gewissenlosen und Rücksichtslosen mit Erfolgen und hohen Gewinnen belohnt. Vor diesem Hintergrund ist zu überlegen, ob man die unumgängliche, ernsthaft zu führende Ethik-Debatte nicht auf eine konzeptionell breitere Basis stellt und mit einer Reflexion des Selbstverständnisses kombiniert. Einen Vorschlag dazu, wie diese Kombination inhaltlich aussehen könnte, habe ich hier skizziert. Natürlich ist mir bewusst, dass der hier angedeutete Weg der Realität der internationalen Rennsportindustrie massiv zuwider läuft und vermutlich zunächst kaum oder keine Anhänger gewinnen wird. Entscheidend ist aber aus meiner Perspektive auch nicht, ob es diese inhaltliche Konzeption sein wird oder eine andere. Entscheidend ist, dass das Wohlergehen des Pferdes bei allen Innovationen im Rennsport, bei allen zu treffenden Maßnahmen und in allen Stadien eines Rennpferdelebens im Vordergrund steht. Die Rennsportgemeinschaft muss sich im klaren darüber sein, dass die natürlichen Grenzen des Pferdes zu respektieren sind; sie muss sich darüber verständigen, was das im einzelnen heißt und sie muss - dies scheint mir das Wichtigste zu sein - offen darüber sprechen, wie schwer es ihr fallen wird, diese Grenzen zu respektieren.

\section{Literatur}

Behnke J. (2013) Entscheidungs- und Spieltheorie; UTB, Stuttgart

Diekmann A. (2009): Spieltheorie. Einführung, Beispiele, Experimente. Rororo, Reinbek

Nussbaum M. (2014) Jenseits von Mitleid und Menschlichkeit - Gerechtigkeit für nichtmenschliche Tiere. In: Schmitz. F. (Hg.) Tierethik. Grundlagentexte; Suhrkamp, Berlin, 176-218 